\title{
Efficacy, Safety and Cost Effectiveness of Metronomic Low Dose Versus Intermittent High Dose Capecitabine in Metastatic Breast Cancer
}

\author{
Hala Elsebaie*(D), Wael Samir Makar Yassa (D), Shaimaa Lasheen (D), Noha Ibrahim (D) \\ Department of Clinical Oncology, Faculty of Medicine, Cairo University, Giza, Egypt
}

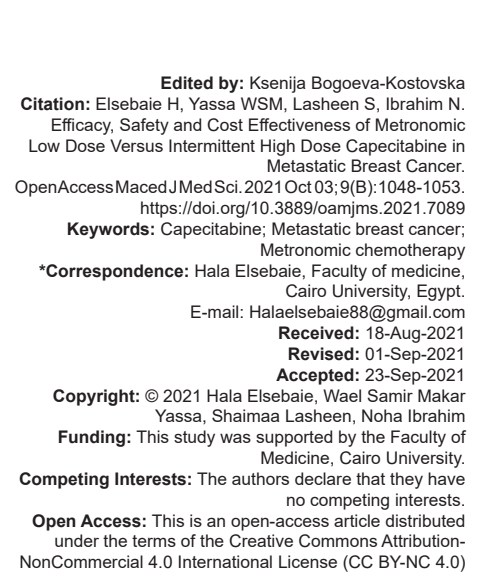

Introduction

Breast cancer is the most common cancer among women worldwide accounting around one in four cancer cases in female population. The estimated age-adjusted annual incidence of breast cancer of $144.9 / 100000$ and mortality of $32.9 / 100000$, with 98755 predicted deaths [1]. Worldwide, there was about 2.1 million new cases diagnosed female breast cancer cases in 2018, accounting for almost one in four cancer cases among women, and $~ 630000$ died of it [2] Through the large incidence the management of metastatic her two negative breast cancer still a matter of debate with many trials about the best chemotherapy regimen and many trials discussed the idea of maintenance treatment. Many studies have shown that maintenance chemotherapy might prolong the time to progression, but few studies have demonstrated an advantage in terms of overall survival (OS). In many cases, toxicities due to the maintenance treatment were not justified by the clinical benefit deriving from the prolongation of therapy. In 1987, in Phase II randomized trial, Coates et al. demonstrated the superiority in terms of clinical response, quality of life, and survival for continuous chemotherapy versus intermittent chemotherapy predicted [1].

Our study is a randomized, parallel assignment study aiming at examining the maintenance treatment of Her2-negative metastatic breast cancer through initial phase of taxotere/capecitabine then randomization to one of two arms both as maintenance capecitabine given in two different doses and schedule to test for the different pattern of toxicity, efficacy, and cost-effectiveness.

\section{Patients and Methods}

\section{Patients}

The protocol described the target population as women with metastatic Her2-negative breast cancer patients who are either de novo metastatic or received previous lines of treatment. Key eligibility criteria include age $\geq 18$ years, histologically confirmed and documented HER2-negative metastatic breast cancer, Patients with at least one measurable lesion according to response evaluation criteria in solid tumors (RECIST) 
Table 1: Baseline tumor characteristics of both groups

\begin{tabular}{|c|c|c|c|c|c|}
\hline \multirow[t]{2}{*}{ Variables } & \multicolumn{2}{|c|}{ Continuous } & \multicolumn{2}{|c|}{ Intermittent } & \multirow[t]{2}{*}{$P$ value } \\
\hline & $n$ & $\%$ & $n$ & $\%$ & \\
\hline \multicolumn{6}{|l|}{ Pathology } \\
\hline IDC & 22 & 84.6 & 21 & 84.0 & \multirow[t]{4}{*}{0.631} \\
\hline ILC & 1 & 3.8 & 1 & 4.0 & \\
\hline MEDULLAR & 0 & 0.0 & 1 & 4.0 & \\
\hline MIXED & 2 & 7.7 & 1 & 4.0 & \\
\hline T3or more & 5 & 19.2 & 10 & 40.0 & 0.248 \\
\hline N3 & 8 & 30.8 & 4 & 16.0 & 0.099 \\
\hline M1 & 10 & 38.5 & 8 & 32.0 & 0.771 \\
\hline Surface area, mean (SD) & 1.80 & $( \pm 0.13)$ & 1.78 & $( \pm 0.17)$ & 0.748 \\
\hline ER Positive & 20 & 76.9 & 18 & 72.0 & 0.874 \\
\hline PR Positive & 16 & 61.5 & 15 & 60.0 & 0.994 \\
\hline \multicolumn{6}{|l|}{ KI67 } \\
\hline High & 10 & 38.5 & 6 & 24.0 & \multirow[t]{2}{*}{0.557} \\
\hline Low & 10 & 38.5 & 9 & 36.0 & \\
\hline \multicolumn{6}{|l|}{ Type of surgery } \\
\hline BCS & 5 & 19.2 & 8 & 32.0 & \multirow[t]{2}{*}{0.501} \\
\hline MRM & 12 & 46.2 & 12 & 48.0 & \\
\hline CA153, mean (SD) & 209.22 & $( \pm 160.35)$ & 157.78 & $( \pm 95.86)$ & 0.302 \\
\hline \multicolumn{6}{|l|}{ Site of METS } \\
\hline Bone & 19 & 73.1 & 17 & 68.0 & 0.691 \\
\hline Lung & 14 & 53.8 & 16 & 64.0 & 0.461 \\
\hline Liver & 15 & 57.7 & 10 & 40.0 & 0.206 \\
\hline \multicolumn{6}{|l|}{ Other Sites of METS } \\
\hline Ascites & 1 & 3.8 & 0 & 0.0 & \multirow[t]{5}{*}{0.316} \\
\hline Bilateral breast & 1 & 3.8 & 0 & 0.0 & \\
\hline BRAIN & 2 & 7.7 & 0 & 0.0 & \\
\hline LNs & 2 & 7.7 & 1 & 4.0 & \\
\hline PLEURA & 2 & 7.7 & 3 & 12.0 & \\
\hline
\end{tabular}

criteria at study entry, Documented ER/PR status. Prior hormone therapy for metastatic disease was allowed but must stop before study entry, Initial performance status by ECOG to be 0,1 and 2 . Life expectancy of $\geq 12$ weeks.

Table 2: Patient's assessment after cycles for both groups

\begin{tabular}{|c|c|c|c|c|c|}
\hline \multirow[t]{2}{*}{ Outcome } & \multicolumn{2}{|c|}{ Continuous } & \multicolumn{2}{|c|}{ Intermittent } & \multirow[t]{2}{*}{$p$ value } \\
\hline & $n$ & $\%$ & $n$ & $\%$ & \\
\hline \multicolumn{6}{|l|}{ Initial phase } \\
\hline Death & 1 & 3.8 & 0 & 0.0 & 0.289 \\
\hline Loss of follow up & 0 & 0 & 0 & 0.0 & \\
\hline Poor tolerance & 0 & 0 & 1 & 4.0 & \\
\hline Progression & 1 & 3.8 & 1 & & \\
\hline Partial response & 17 & 69.2 & 15 & 60.0 & \\
\hline Stationary & 6 & 23 & 8 & 32.0 & \\
\hline $\begin{array}{l}\text { Complete } \\
\text { response }\end{array}$ & 1 & 3.8 & 0 & 0 & 0.2 \\
\hline \multicolumn{6}{|l|}{ Maintenance phase } \\
\hline $\begin{array}{l}\text { Complete } \\
\text { response }\end{array}$ & 1 & 4 & 0 & 0 & 0.3 \\
\hline Partial response & 12 & 50 & 4 & 16.6 & 0.038 \\
\hline $\begin{array}{l}\text { Stationary } \\
\text { disease }\end{array}$ & 4 & 16 & 5 & 21 & \\
\hline $\begin{array}{l}\text { Progressive } \\
\text { disease }\end{array}$ & 7 & 29 & 14 & 58 & 0.04 \\
\hline Death & 0 & 0 & 1 & 4 & 0.3 \\
\hline
\end{tabular}

radiotherapy for the treatment of metastatic disease or major surgical procedure within 28 days prior to the first study treatment. Unsuitability for chemotherapy with either inadequate bone marrow function: absolute neutrophil count: $<1.5 \times 10^{9} / \mathrm{L}$, platelet count $<75 \times 10^{9} / \mathrm{L}$ or hemoglobin $<100 \mathrm{~g} / \mathrm{L}$, Inadequate liver or renal function with total bilirubin $>2$ ULN, AST/ALT $>2.5$ ULN, serum creatinine $>1.58 \mathrm{mg} / \mathrm{dl}$. Pregnant or lactating females, Her-2 positive (ICH +++ or FISH positive), Concurrent or within 30 days using drugs of other clinical trials, Previous treatments containing Capecitabine (whether adjuvant or palliative treatment). The human research ethics review committee at the faculty of medicine, Cairo University, approved the protocol. Random assignment was performed. Participants were randomly assigned to intermittent capecitabine or continuous capecitabine.
Table 3: Cost-effective analysis for each group

\begin{tabular}{lll}
\hline Study arms & Continuous & Intermittent \\
\hline Cost for the treatment course & $2950 \$$ & $3783 \$$ \\
Total cost after all cycles & $53107 \$$ & $68085 \$$ \\
Cost per responding case & $727 \$$ & $1135 \$$ \\
\hline
\end{tabular}

\section{Treatments}

All patients received Capecitabine (1000 mg/ $\mathrm{m}^{2}$ twice daily D1-14 Q3W) plus docetaxel (75 mg/m2, D1,Q3W) for a maximum of 6 cycles, or be treated until disease progression, unacceptable toxicity or patient request for withdrawal, whichever occurs first. Each cycle is 3 weeks in duration for the patients with SD, PR, or CR after the initial treatment phase will enter into the maintenance treatment phase, Randomization every other eligible patient to one of the two arms. Experimental: Metronomic Capecitabine $650 \mathrm{mg} / \mathrm{m}^{2}$ twice per day on days 1-21 of each 3-week cycle. Active Comparator: Intermittent Capecitabine $1000 \mathrm{mg} / \mathrm{m}^{2}$ twice daily on days 1-14 of each 3-week cycle. Doses were based on the surface area. Treatment was delayed until toxicities other than alopecia had resolved to Grade 1 or lower. Dose reductions according to toxicity were performed. Treatment was continued until disease progression, patient intolerance, or unacceptable toxicity without any specified maximum duration. Management after disease progression was according to opinion of our committee.

Table 4: Grade 3 and 4 toxicities in both arms

\begin{tabular}{|c|c|c|c|c|c|}
\hline \multicolumn{6}{|c|}{ Table adverse events of maintenance treatment } \\
\hline \multirow{2}{*}{$\begin{array}{l}\text { Study arms } \\
\text { Adverse events }\end{array}$} & \multicolumn{2}{|l|}{ Intermittent } & \multicolumn{2}{|l|}{ Continous } & \multirow[t]{2}{*}{$p$ value } \\
\hline & Grade 1-2 & Grade 3-4 & Grade 1-2 & Grade 3-4 & \\
\hline Nausea & $17(70 \%)$ & $5(20 \%)$ & $9(37.5)$ & 0 & 0.03 \\
\hline Vomiting & $15(62 \%)$ & $4(16 \%)$ & $8(33.3 \%)$ & $1(4.1 \%)$ & 0.045 \\
\hline Diarrhea & $14(58 \%)$ & $1(4.1 \%)$ & $7(29 \%)$ & 0 & 0.046 \\
\hline Hand -foot syndrome & $13(54 \%)$ & $4(16.6 \%)$ & $8(33.3 \%)$ & $1(4.1 \%)$ & 0.051 \\
\hline Alopecia & $2(8.3 \%)$ & 0 & 0 & 0 & 0.5 \\
\hline Neutropenia & $5(20.8)$ & $1(4.1 \%)$ & $1(4.1 \%)$ & 0 & 0.048 \\
\hline Anemia & $7(29 \%)$ & $2(8.3 \%)$ & $5(20.8 \%)$ & 0 & 0.6 \\
\hline Transaminitis & $3(12.5 \%)$ & $1(4.1 \%)$ & 0 & 0 & 0.06 \\
\hline Neuropathy & $5(20.3 \%)$ & $1(4.1 \%)$ & $4(16.6 \%)$ & 0 & 0.8 \\
\hline DVT & 0 & 0 & 0 & $1(4.1 \%)$ & 0.9 \\
\hline
\end{tabular}

\section{Assessments}

Patients were assessed every 3 weeks before the start of the new cycle with history, clinical examination, and routine laboratories complete blood count, liver function tests, renal function tests, and tumor markers. Adverse events were rated according to the National Cancer Institute Common Toxicity Criteria, version 4.0. Computerized axial tomographic scans of the chest and abdomen and skeletal radionuclide scans were performed at baseline and every 12 weeks until progression.

Primary endpoint was the assessment of side effects and toxicity profiling. Laboratory test graded according to the NCI CTCAE (version 4.0), premature withdrawals, and vital signs. Hand-foot syndrome and diarrhea will be especially interested, adverse events of special interest: hand-foot syndrome and diarrhea. Secondary endpoint was progression free survival (PFS, 
from time of randomization to disease progression), OS (OS, from randomization to death), clinical benefit rate $(C B R$, including patients with $C R, P R, S D$ after 6 months of treatment), overall response rate (ORR, including patients with $\mathrm{PR}, \mathrm{CR}$ ) and cost-effectiveness was calculated by dividing the total cost by the outcome. Objective tumor response was assessed according to RECIST 1.0.

\section{Statistical considerations}

The planned sample size of 51 patients was recruited over 2.5 years and followed for additional 1 year comparing between the two arms of maintenance treatment with intermittent or continuous Capecitabine. Kaplan-Meier curves for PFS and OS were constructed and compared using the log-rank test for the primary analyses of time to event data. The influence of baseline factors on treatment effects was assessed by testing for interactions with treatment in Cox proportional hazards models for PFS and OS.

\section{Results}

The current study included 51 patients with breast cancer were included between June 2017 and January 2020. 26 patients were randomized to receive continuous capecitabine regimen and 25 patients receive intermittent capecitabine regimen after the initial combination therapy phase (Figure 1).

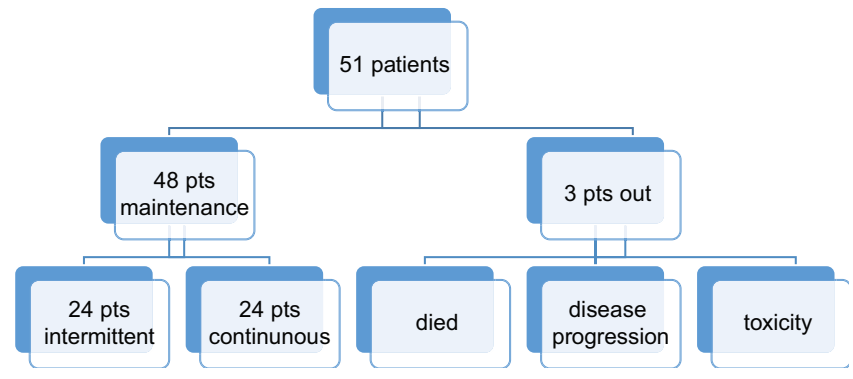

Figure 1: Diagram of study patients

During combination therapy one patient died as a complication of uncontrolled diabetes, one patient had severe neuropathy Grade 4 and did not receive $6^{\text {th }}$ cycles Taxotere and proceeded to metronomic continuous capecitabine after supportive treatment and one patient had progressive disease and liver cell failure and didn't continue maintenance treatment, also one patient had poor tolerance to treatment and lost follow up after the $4^{\text {th }}$ cycle. Among the 51 patients, 48 patients proceeded to maintenance treatment phase 24 patients in each arm.

Baseline characteristics between both groups mean age of $54.08( \pm 9.5), 57.7 \%$ of cases were postmenopausal, $15.4 \%$ were diabetic, $26.9 \%$ were

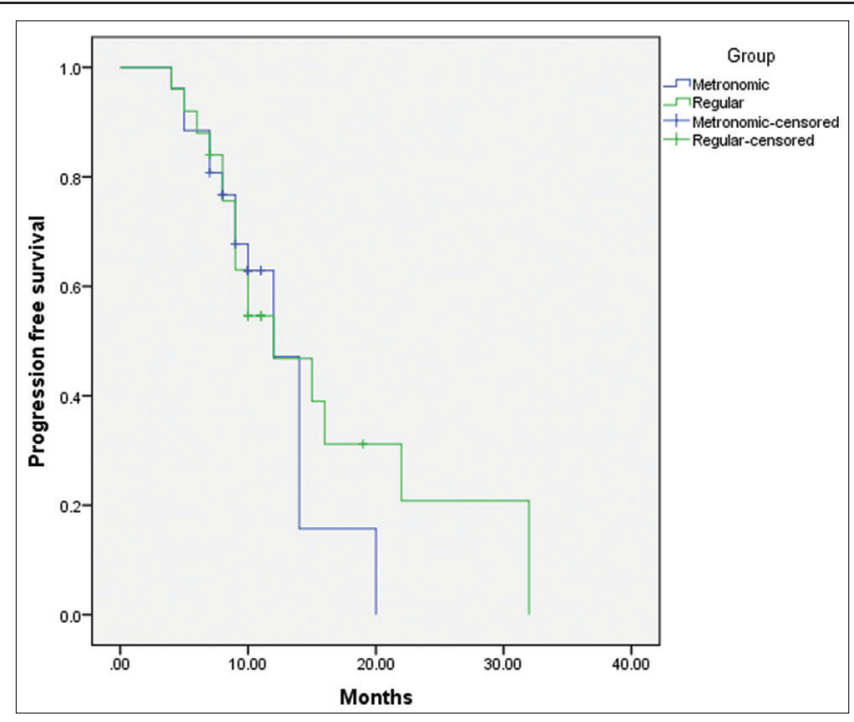

Figure 2: Kaplan Meier curve showing progression free survival in each group

hypertensive in the continuous capecitabine group with performance status, $53.8 \%$ had score 1 and $46.2 \%$ had score two. While in the intermittent capecitabine group mean age was 47.67 ( \pm 8.84$), 76.0 \%$ of cases were premenopausal, only one case was diabetic, two cases were hypertensive with performance status, $60.0 \%$ had score 1 and $40.0 \%$ had score two (Table 1 ).

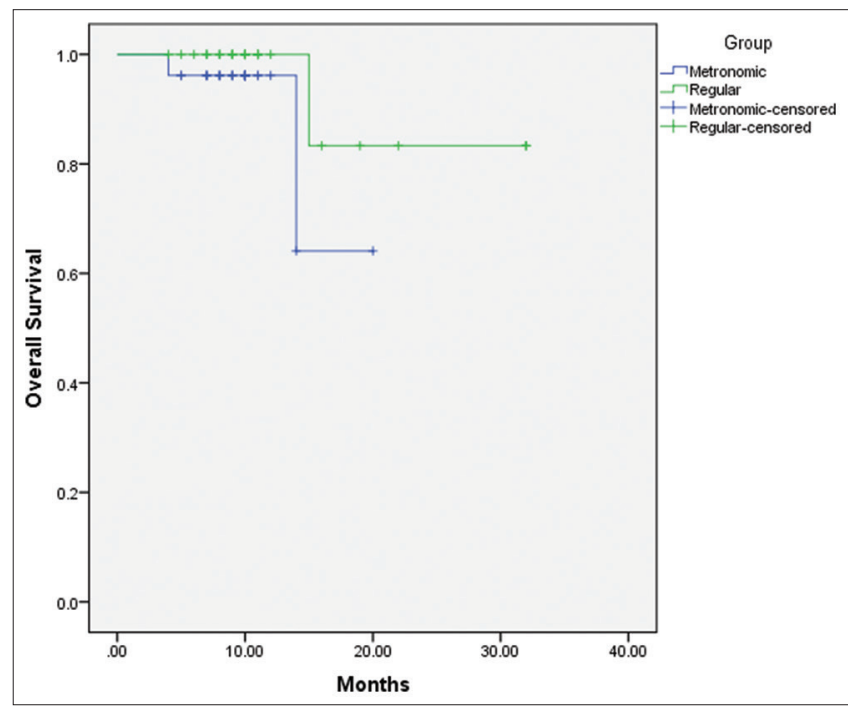

Figure 3: Kaplan Meier curve showing overall survival in each group

There was significant different between two groups regarding; age $(p=0.018)$ and menopausal state $(p=0.015)$. In the continuous capecitabine arm, $73.3 \%$ had bone metastasis while $57.7 \%$ had liver metastasis while in the intermittent capecitabine arm $68 \%$ had bone metastasis while $40 \%$ had liver metastasis. Most of the cases received the initial regimen Taxotere/capecitabine as first-line treatment $(61.5 \%$ in the continuous capecitabine arm while $40 \%$ in the intermittent capecitabine arm). While in the adjuvant, sitting more than half of cases received hormonal tamoxifen and chemotherapy anthracyclines with taxanes. 


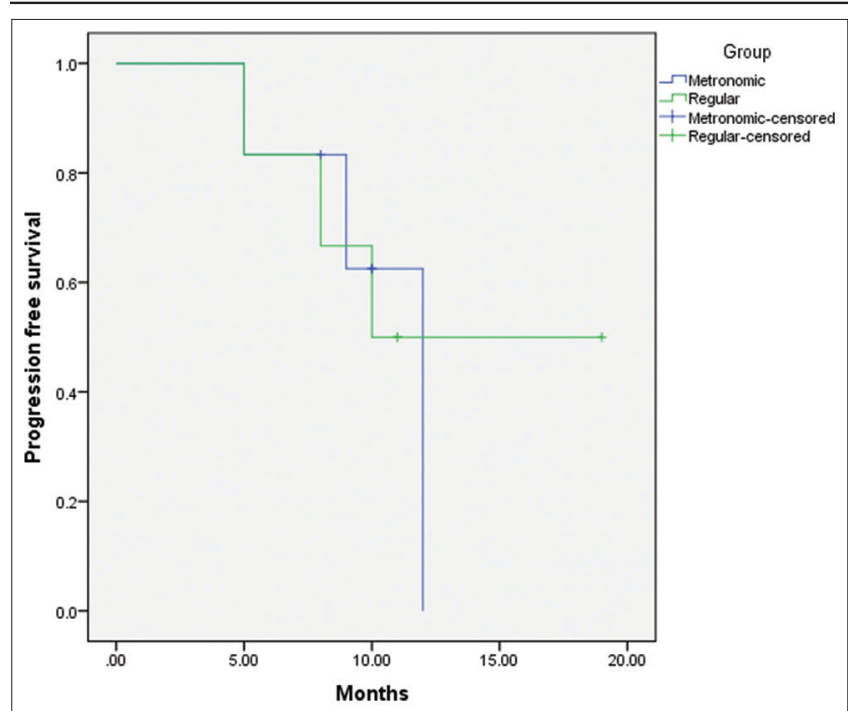

Figure 4: Kaplan Meier curve showing progression free survival for triple negative patient in each group

Objective tumor response in both arms $66 \%$ had disease control at 6 months on treatment in the continuous capecitabine arm, and $37 \%$ had control in the intermittent capecitabine arm. Progressive disease was documented in $29 \%$ in the continuous capecitabine arm, while it was $58 \%$ in the intermittent capecitabine arm (Table 2).

Cost-effectiveness: In each cycle, cost was 2950 \$ in continuous capecitabine arm versus 3783 \$ in intermittent capecitabine arm. Estimated total cost after all cycles; 53107 \$ in METRONOMIC group versus $68085 \$$ in regular group (Table 3).

The estimated cost to have a responding case was $727 \$$ in continuous capecitabine arm versus $1135 \$$ in intermittent capecitabine arm with nearly $35.9 \%$ reduction of cost in continuous capecitabine arm than intermittent capecitabine so the continuous arm was more cost-effective.

Mean PFS was higher in intermittent
capecitabine than continuous capecitabine group (15.8 months vs. 12.1 months, Log-rank: $p=0.656$ ). Furthermore, mean OS was higher in the intermittent arm than the continuous arm group (29.9 months vs. 17.4 months, Log-rank: $p=0.188$ ) (Figures 2 and 3 ).

\section{The toxicity}

The number of Grade 3 and 4 toxicities were higher in the intermittent capecitabine arm significantly than the continuous arm, there were higher number of Grade 3 and 4 toxicity of nausea and vomiting in the intermittent capecitabine arm with significant $p=0.03$ and 0.045 respectively. Also, regarding diarrhea, the number of patients suffering from Grade 3 and 4 toxicity was significantly higher in the intermittent arm with significant $p=0.046$ while hand and foot syndrome the number of cases was higher in the intermittent arm with $p=0.051$. Furthermore, there was higher incidence of neutropenia with fever in the intermittent arm vs the continuous arm with a significant $p=0.048$. There was only one patient in the continuous capecitabine arm who had arm deep vein thrombosis. Several patients suffered from anemia but only two pts in the intermittent arm had G 3 and 4 anemia and required blood transfusion with insignificant $p$ value (Table 4).

The total number of cycles given to patients was 731 between the initial phase Taxotere/capecitabine and maintenance capecitabine given in intermittent and continuous regimens. The median number of cycles in the continuous capecitabine arm was 18 (range 3-22), while than median number of cycles in the intermittent capecitabine arm was 13 (range 3-22) with significant $p=0.031$.

We conducted a survival analysis for patients with ER - PR negative receptors. In triple negative cases, mean PFS was 13.3 months in intermittent group and 10.2 months in continuous group (Log-rank: $p=0.953)($ Figure 4).

\section{Discussion}

The idea of maintenance Capecitabine had been discussed in the metastatic setting in several studies such as In Surmeli et al., 2015. The present study included 55 metastatic breast cancer patients with HER2-negative disease. They received Docetaxel/Capecitabine regimen. During combination chemotherapy one patient died, one patient was lost to follow-up and one patient developed the intolerable hand-foot syndrome. Response assessments were made for 52 patients. After 6 cycles of combination chemotherapy 29/52, (55.7\%) patients had PR, $3 / 52(5.8 \%)$ patients had CR and 16/52(30.8\%) patients had stable disease. After the initial combination chemotherapy, responding patients were kept on maintenance Capecitabine. The median PFS and OS were 5.5, 26.6 months. The maintenance therapy improved response in $8.3 \%$ (2PR, 2CR) with $66.7 \% \mathrm{SD}$. The median number of maintenance therapy was 6.5 [3]. In the present study, the PFS was higher 13.3 months and the improved response was $16.6 \%$. This may be due to less incidence of visceral metastasis $(54 \%)$ and fewer number of tumor sites.

In a meta-analysis [4], 22 trials, Metronomic Capecitabine was studied in 11 trials. The CBR of MCT was $55.6 \%$ and the PFS at 6 months was $56.8 \%$. The OS at 12 and 24 months was $70.3 \%$ and $40 \%$. There was no statistical significance in treatment outcome in combination regimens. MCT showed less toxicity (Liu et al., 2017). The CBR in the present study was higher than the metaanalysis $(73.1 \%$ and $64 \%$ in the continuous and intermittent group respectively). This 
may be due to the exclusion of Her2 positive cases. In the meta-analysis of [4] ORR data was extracted from 17 trials for this meta-analysis. The pooled ORR was $34.1 \%(95 \% \mathrm{Cl} 27.4-41.5)$ by using the random effects model (heterogeneity analysis: $Q=67.5, P 5$, $p=0.001$ ), subgroup analysis based on whether MCT was used alone or combined with other drug therapies. There was no statistically significant difference in the ORR between mast cell tumours (MCT) used alone and the combination schemes $(33.5 \%$ vs. $34.2 \%$, respectively, $p=0.925$ ).

In our study the triple negative breast cancer (TNBC) patients were $25.4 \%$, mean PFS was 13.3 months in intermittent Capecitabine arm and 10.2 months in continuous Capecitabine arm with no significant difference $(p=0.953)$. TNBC receiving treatment as first line in the metastatic setting had a better clinical response $(p=0.07)$. While in the study done by Ozdemir et al. 2013, the triple-negative patient response rate was $28.1 \%$; there was no significant difference between ER and PR positive groups compared to triple-negative group in terms of PFS and OS (7 vs. 8 months, and 14 vs. 17 months, respectively) [5] that Stockler et al. 2011 found longer survival was associated with the presence of hormone receptors positive [6].

In China, a study was conducted in 265 metastatic TNBC exhibiting disease control after firstline platinum-based chemotherapy. Maintenance treatment was given to158 patients and 107 were kept on follow-up. The median PFS in the maintenance was 9.63 versus 7.47 months in the observation group $(p<0.0001)$. The median OS was 25.37 versus 31.27 month [7]. In our study median PFS was 13.3 months and the median OS was 9.9 months due to short period of follow-up. In a study by Cazzaniga et al., 2016 [8], the PFS was 4.7 months [8]. This is shorter than our study may be because the median age in the TNBC group were older, 69 years (47-85). The majority of patients had visceral involvement at the time of enrolment $(23,82.1 \%)$ with only $4(14.8 \%)$ having $<2$ sites of metastasis.

Regarding cost-effectiveness, in our study, each cycle cost was 2950 \$ and 3783 \$ in the continuous and the intermittent group respectively. Estimated total cost after all cycles was lower in the continuous group; 53107 \$ versus $\$ 68085 \$$. The estimated cost to have a responding case was $727 \$$ versus $1135 \$$ in the continuous versus the intermittent Capecitabine arm with nearly $35 \%$ reduction of cost in continuous arm. Hence, the continuous Capecitabine arm was more cost-effective.

While in the study of Abdallah et al., 2017 [9] the low dose Capecitabine was compared to Exemestane regarding cost-effectiveness. They found that the total cost for the Exemestane group was lower than the Capecitabine group $(1,699,087$ EGP vs 2,389,345 EGP, respectively) with a net difference of 690258 EGP.
These results showed that Exemestane provides better quality-adjusted life-year (QALYS) at lower costs compared to Capecitabine.

The present study was limited by the small number of patients and short time of follow up. There was difficulty in recruitment to the COVID19 pandemic. Furthermore, there was delay in the regular imaging and tumor marker in the routine assessment.

We conclude that metronomic continuous capecitabine is well tolerable and less toxic than intermittent and proved to be more cost-effective. The clinical response was significantly better in patients with metastatic TNBC and those receiving metronomic chemotherapy on the setting of first-line treatment.

\section{Conclusion}

We conclude that metronomic continuous capecitabine is well tolerable and less toxic than intermittent and proved to be more cost-effective. The clinical response was significantly better in patients with metastatic TNBC and those receiving metronomic chemotherapy on the setting of first-line treatment.

\section{References}

1. Grilli R, Chiesa V. Overuse in cancer care: Do European studies provide information useful to support policies? Health Res Policy Syst. 2018;16(1):12. https://doi.org/10.1186/s12961-018-0287-z PMid:29458403

2. Bray F, Ferlay J, Soerjomataram I, Siegel RL, Torre LA, Jemal A. Global cancer statistics 2018: GLOBOCAN estimates of incidence and mortality worldwide for 36 cancers in 185 countries. CA Cancer J Clin. 2018;68(6):394-424. https://doi. org/10.3322/caac.21492

PMid:30207593

3. Surmeli ZG, Varol U, Cakar B, Degirmenci M, Arslan C, Piskin GD, et al. Capecitabine maintenance therapy following docetaxel/capecitabine combination treatment in patients with metastatic breast cancer. Oncol Lett. 2015;10(4):2598-602. https://doi.org/10.3892/ol.2015.3546

PMid:26622896

4. Liu Y, Gu F, Liang J, Dai X, Wan C, Hong X, et al. The efficacy and toxicity profile of metronomic chemotherapy for metastatic breast cancer: A meta-analysis. PLoS One. 2017;12(3):e0173693. https://doi.org/10.1371/journal.pone.0173693

PMid:28296916

5. Ozdemir N, Aksoy S, Sendur MA, Akinci MB, Yazici O, Budakoglu B, etal. Capecitabine/cisplatin doublet in anthracycline and taxane pretreated and HER-2 negative metastatic breast carcinoma patients. J BUON. 2013;18(4):831-7. PMid:24344005

6. Stockler MR, Harvey VJ, Francis PA, Byrne MJ, Ackland SP, Fitzharris $\mathrm{B}$, et al. Capecitabine versus classical 
cyclophosphamide, methotrexate, and fluorouracil as firstline chemotherapy for advanced breast cancer. J Clin Oncol. 2011;29(34):4498-504. https://doi.org/10.1016/j. breastdis.2012.06.030

PMid:22025143

7. Chen Y, Zhang J, Hu XC, Wang BY, Wang ZH, Wang LP, et al. Maintenance chemotherapy is effective in patients with metastatic triple negative breast cancer after first-line platinumbased chemotherapy. Ann Palliat Med. 2020;9(5):3018-27. https://doi.org/10.1093/annonc/mdz242.058

PMid:32787354

8. Cazzaniga ME, Cortesi L, Ferzi A, Scaltriti L, Cicchiello F,
Ciccarese $\mathrm{M}$, et al. Metronomic chemotherapy with oral vinorelbine (mVNR) and capecitabine (mCAPE) in advanced HER2-negative breast cancer patients: Is it a way to optimize disease control? Final results of the VICTOR-2 study. Breast Cancer Res Treat. 2016;160(3):501-9. https://doi.org/10.1007/ s10549-016-4009-3

PMid:27752847

9. Abdallah H, Abourawash AS, Elgazzar M, Helal A. PCN124cost effectiveness analysis of exemestane versus capecitabine monotherapy for patients with hormone receptor-positive and her2-negative, metastatic breast cancer from national cancer institute prespective in Egypt. Value Health. 2017;20(9):A434. https://doi.org/10.1016/j.jval.2017.08.207 